\title{
Video Article \\ Isolation of Tissue Extracellular Vesicles from the Liver
}

\author{
Kaori Ishiguro ${ }^{1}$, Irene K. Yan ${ }^{1}$, Tushar Patel ${ }^{1}$ \\ ${ }^{1}$ Department of Transplantation, Mayo Clinic \\ Correspondence to: Tushar Patel at patel.tushar@mayo.edu
}

URL: https://www.jove.com/video/58649

DOI: doi:10.3791/58649

Keywords: Biochemistry, Issue 150, Liver, extracellular vesicles, collagenase, ultra-centrifugation, diagnostics, perfusion

Date Published: 8/21/2019

Citation: Ishiguro, K., Yan, I.K., Patel, T. Isolation of Tissue Extracellular Vesicles from the Liver. J. Vis. Exp. (150), e58649, doi:10.3791/58649

(2019).

\section{Abstract}

Extracellular vesicles (EVs) can be released from many different cell types and detected in most, if not all, body fluids. EVs can participate in cell-to-cell communication by shuttling bioactive molecules such as RNA or protein from one cell to another. Most studies of EVs have been performed in cell culture models or in EVs isolated from body fluids. There is emerging interest in the isolation of EVs from tissues to study their contribution to physiological processes and how they are altered in disease. The isolation of EVs with sufficient yield from tissues is technically challenging because of the need for tissue dissociation without cellular damage. This method describes a procedure for the isolation of EVs from mouse liver tissue. The method involves a two-step process starting with in situ collagenase digestion followed by differential ultra-centrifugation. Tissue perfusion using collagenase provides an advantage over mechanical cutting or homogenization of liver tissue due to its increased yield of obtained EVs. The use of this two-step process to isolate EVs from the liver will be useful for the study of tissue EVs.

\section{Video Link}

The video component of this article can be found at https://www.jove.com/video/58649/

\section{Introduction}

Extracellular vesicles (EVs) are membrane-bound vesicles that are released from many different types of cells in the body. EVs contain a cargo of molecules that include RNA, DNA, and protein. Transfer of this cargo by EVs from one cell to another is postulated as one mechanism by which cells within tissues communicate with each other ${ }^{1}$. The majority of information regarding the cargo or roles of EVs in normal health and diseases has been derived from studies on EVs obtained from cells in culture or collected from the circulation or other body fluids ${ }^{2}$. In order to understand their physiological roles in vivo, a robust method is necessary for the isolation of tissue EVs that captures all populations of EVs and avoids cellular damage or contamination ${ }^{3}$. The overall goal of the method described herein is to isolate tissue EVs from mouse livers.

Most cell types in the liver have been shown to produce EVs, and the study of EV-based signaling is advancing basic knowledge and understanding of hepatic diseases. However, the combined impact of EVs from different cell types within tissues is only partially understood. Isolation of EVs from liver tissues is necessary in order to understand the in situ contributions of EVs within the tissue milieu. The approach described herein is based on a two-step perfusion to enhance tissue dissociation and minimize cell damage. Subsequently, EVs are isolated from the dissociated liver tissue. Approaches using two-step perfusion for isolation of hepatocytes have been used since the early $1950 \mathrm{~s}^{4}$. These methods for hepatocyte isolation have been modified and continuously improved and are now standard approaches for isolation of hepatocytes in cultures, in cell suspensions, and from tissues ${ }^{5,6,7}$. In the first step, the liver is subjected to a non-recirculating perfusion with calcium-free buffer,Hank's balanced salt solution (HBSS). In the second step, the liver is perfused with collagenase to dissolve the extracellular matrix for further separation of desmosomal cell-to-cell junctions. An optimal treatment time for collagenase dissolution is 7 to 10 minutes. A shorter duration of treatment will cause incomplete dissolution and retain cell contacts in the liver, whereas a longer duration may cause liver damage or portal vein disruption. EVs are then isolated using differential centrifugation to remove cells and cellular debris. This results in EV collection in high yields that can be used for further downstream analyses or studies.

\section{Protocol}

All studies involving animals were performed in accordance with a protocol that was approved by the Mayo Clinic Institutional Animal Care and Use Committee.

\section{Bench Preparation}

1. Prepare a water bath and set it to $37^{\circ} \mathrm{C}$. The other necessary equipment and set-up are shown in Figure 1.

2. Measure $100 \mathrm{mg}$ of collagenase type IV and add it to a $125 \mathrm{~mL}$ flask containing $100 \mathrm{~mL}$ of HBSS in a water bath (40 $\left.{ }^{\circ} \mathrm{C}\right)$. Ensure that the collagenase has been dissolved by swirling the liquid in the flask, and also make sure that the flask is submerged completely in the water bath. For greater efficiency, allow the collagenase to dissolve for at least $30 \mathrm{~min}$ even though it may appear to dissolve instantly. 
3. Submerge a $125 \mathrm{~mL}$ flask containing $50 \mathrm{~mL}$ of HBSS in a water bath at $40{ }^{\circ} \mathrm{C}$. This will be used for initial flushing.

4. Spray down the surface of all instruments such as the scissors and forceps with $70 \%$ ethanol. Prepare a few clean cotton swabs.

5. Rinse out the tubing of the pump by running $70 \%$ ethanol through the pump and remove any residual ethanol by rinsing it twice with running water.

6. Lay an absorbent bench pad on the lab bench and place a hard box container on top. This will be needed to contain any excess liquids during the mouse perfusion. Wrap a polystyrene foam pad with aluminum foil and place it inside the container.

7. Place a sterile $10 \mathrm{~cm}$ culture dish close to the bench. This will be used to hold the digested liver after the perfusion is completed.

8. Pour $10 \mathrm{~mL}$ of collagenase medium (step 1.2) into a sterile culture dish in advance.

9. Using a winged blood collection set, connect the 23-gauge to the free end of the pump tubing (cutting the wings off the butterfly cannula can lead to better handling). Pass until the tip of the cannula of blood collection is filled.

\section{Animal Preparation}

1. Before starting anesthesia using isoflurane, make sure that an adequate amount of supply gas is available for the duration of the procedure. Turn on the oxygen $\left(\mathrm{O}_{2}\right)$ supply to the induction chamber at $1-2 \mathrm{~L}$, then turn on isoflurane between $2-4 \%$ using a flowmeter.

2. Put the mouse into the induction chamber and close the top door. Monitor the mouse until it is recumbent. NOTE: The gases in the chamber will keep the mice anesthetized for several minutes.

3. Place the mouse on a polystyrene foam pad wrapped with aluminum foil. Switch the flow from the induction chamber to a nosecone. Ensure that anesthesia is adequate. If the mouse has started responding, gently restrain it in a nose cone until the it is fully anesthetized.

4. Ensure anesthesia by monitoring respiration and response to stimulation during the procedure. Adjust the rate flowmeter as needed to ensure adequate anesthesia. The paws must be nonresponsive to the pinch test under anesthesia. Additional details may be obtained from the laboratory guide for the care of animals.

5. Tape or pin down all four limbs of the mouse.

6. Clean the skin over the abdomen by spraying with $70 \%$ ethanol and wiping it off with gauze and an alcohol pad. This step is critical to avoid contamination from the mouse fur.

7. Make a wide-open cut through the skin from the anterior to the pelvis bone using a set of sterile scissors. Be careful not to cut any internal organs . Displace the intestines to the animal's left side by using a cotton tipped applicator to gently to expose the portal vein (PV) and inferior vena cava (IVC).

\section{Cannulation and Perfusion (0.5 h)}

1. Using curved forceps, place a thread underneath the portal vein and tie a knot loosely to prepare cinching tightly after cannulation.

2. Insert the cannula ( $23 \mathrm{G}$ from the blood collection set) into the portal vein 5-10 mm below the ligature. Do not insert the cannula past the first portal branch, otherwise the right anterior lobe can be inadequately perfused. The cannula can be fixed or fastened with thread using a stopper knot.

3. Start the pump to infuse in HBSS at a low flow rate $(1-2 \mathrm{~mL} / \mathrm{min})$. Once the cannulation is confirmed to be successful, the liver will begin to blanch.

4. Cut the IVC to relieve pressure and allow excessive fluid within the liver to drain. This is best performed using the operators other hand so the cannula is not moved.

5. Slowly increase the flow rate to $8 \mathrm{~mL} / \mathrm{min}$ and complete the perfusion using the entire $50 \mathrm{~mL}$ volume of HBSS through the liver, over the next 5 min.

6. Change the collagenase containing medium (step 1.2) into the beaker just before the HBSS is starting to run out. Ensure that air bubbles are not present and do not flow into the liver when changing the medium.

7. Apply transient pressure to the IVC at $5 \mathrm{~s}$ intervals by clamping with forceps. This will cause the liver to swell and help with tissue digestion and dissociation (7-8 $\mathrm{min}$ ). As digestion progresses, the liver will swell and become white. The liver can swell uniformly to approximately twice its original size.

8. Turn off the pump and remove the cannula once digestion is completed. The completion of liver digestion will depend on the size of the mouse and condition of the liver. A dent in the liver can be observed if a cotton-tipped applicator is used to gently probe the liver.

9. Remove the gallbladder from the liver, being careful not to tear it. Using a washed pair of scissors and forceps, extract the liver from the mouse into a sterile $10 \mathrm{~cm}$ culture dish containing phosphate-buffered saline (PBS) for surface washing. Transfer the liver carefully into the sterile $10 \mathrm{~cm}$ culture dish containing collagenase medium (from step 1.8). This is a critical step for avoiding contamination from the mouse blood and bile.

10. Grab and tear apart the liver with two clean forceps while gently shaking the cells from the liver. As this happens, the medium will become clouded. All hepatocytes can be shaken away, leaving behind the connective tissue and vascular tissue.

11. Triturate the cell solution many times using a $3 \mathrm{~mL}$ syringe until the undigested parts of the liver are shaken off. Pour it off into a $50 \mathrm{~mL}$ conical tube with $70 \mu \mathrm{m}$ nylon cell strainers to filter any undigested connective tissue. Wash the dish with HBSS to collect the remaining cells and fill up the $50 \mathrm{~mL}$ conical tube.

12. Centrifuge softly the $50 \mathrm{~mL}$ conical tube in a swinging bucket rotor at $50 \mathrm{xg}$ for $10 \mathrm{~min}$ at $4{ }^{\circ} \mathrm{C}$.

13. Transfer the supernatant to a new $50 \mathrm{~mL}$ conical tube.

\section{Isolation of EVs (5 h)}

1. Centrifuge the supernatant at $300 \times \mathrm{g}$ for $10 \mathrm{~min}$ at $4{ }^{\circ} \mathrm{C}$. Transfer the supernatant into a new $50 \mathrm{~mL}$ conical tube.

2. Centrifuge the supernatant at $2000 \times \mathrm{g}$ for $20 \mathrm{~min}$ at $4{ }^{\circ} \mathrm{C}$ to remove cell debris and aggregates.

3. Transfer the supernatant to a round-bottom tube and centrifuge the supernatant at $10,000 \times \mathrm{g}$ for $70 \mathrm{~min}$ at $4^{\circ} \mathrm{C}$.

4. Collect the supernatant and place into a polycarbonate ultracentrifuge tube and centrifuge at $100,000 \times \mathrm{g}$ for $70 \mathrm{~min}$ at $4{ }^{\circ} \mathrm{C}$.

5. Collect the pellet in an ultracentrifuge tube which is then washed by re-suspending in PBS. Centrifuge the supernatant further at $100,000 \times g$ for $70 \mathrm{~min}$ at $4{ }^{\circ} \mathrm{C}$. 
6. The final pellet comprising of cellular nanovesicles can be directly used for experiments or re-suspended with $1000 \mu \mathrm{L}$ of PBS and stored at $-80^{\circ} \mathrm{C}$.

\section{Assessment of Quality and Yield of Isolations}

1. Assess the size distribution and concentration using nanoparticle tracking analysis or tunable resistive pulse sensing following the instrument manufacturer's protocols.

2. Perform further isolation and purification of specific vesicle populations by various approaches such as the addition of a sucrose gradient or cushion, immunoaffinity techniques, or size exclusion chromatography, based on specific experimental needs.

\section{Representative Results}

The apparatus needed for these isolations comprises of standard laboratory equipment, making this a relatively simple and cost-effective approach. Isolations have been performed from twelve- to thirty-week-old male and female Balb/c or FVB mice. The tray holding the mouse is lined with aluminum foil inside a hard-walled container that collects excess fluids during the perfusion. The flasks containing HBSS or collagenase-containing medium are submerged in a water bath $\left(40^{\circ} \mathrm{C}\right)$ ready to be used. Two sterile $10 \mathrm{~cm}$ culture dishes are used. One is needed for surface washing with PBS, and the other for hepatocyte separation from the connective tissue components.

In this method, the liver is perfused in a non-continuous manner via the portal vein in preference to cannulation from the inferior vena cava. An alternative and commonly used perfusion approach is to perform retrograde perfusion by cannulating the inferior vena cava and cutting the portal vein for drainage. However, portal vein cannulation is easy to access and involves a short distance to the liver, as the portal vein feeds directly into the liver ${ }^{8}$. The selection of an insertion point for cannulation is crucial for optimal success (Figure 2). The cannula is placed past the branches of the stomach and pancreatic veins but not beyond the first portal branch (right and left hepatic portal veins). Once the optimal insertion location in the portal vein is identified, curved forceps are used to place a thread underneath portal vein and tie a loose knot. The needle of cannula is fixed with thread using a stopper knot to stop the needle from falling out.

Figure 3 outlines the overall processing scheme for the differential centrifugation for liver tissue EVs isolation. Ultracentrifugation removes cells, debris and other impurities. The first four centrifugation steps $(50 \mathrm{x} \mathrm{g}, 300 \times \mathrm{g}, 2,000 \mathrm{xg}, 10,000 \mathrm{x} \mathrm{g})$ are designed to remove hepatocytes, intact other cells, dead cells, or cell debris respectively. (Figures 4A and 4B). After these steps, ultracentrifugation is again performed at $100,000 \times g$ to collect the pellet (Figure 4C). The pellet is washed by re-suspending in PBS and subjected to a final ultracentrifugation at $100,000 \times \mathrm{g}$. The pellet after ultracentrifugation is absolutely visible and viscid in this procedure compared to EVs from conditioned culture media. Pipetting many times is required until any brown aggregates are out of sight and completely dissolved. The final pellet is re-suspended with $1000 \mu \mathrm{L}$ of PBS (Figure 4D). Ultracentrifugation removes impurities and other soluble contaminants from the plasma, which can affect functional experimental outcomes. The centrifugation is be carried out at $4^{\circ} \mathrm{C}$.

From mouse liver, this method yields a tissue EV concentration that ranges from 1.74 to $4.00 \times 10^{12}$ with a mean of $3.46 \times 10^{12}$ particles per mL as determined by nanoparticle tracking analysis (NTA) (Figure 5). The mean size of the isolated liver tissue EVs was $157.7 \mathrm{~nm}$, with a mode size of $144.5 \mathrm{~nm}$ and EV sizes ranging from $100-600 \mathrm{~nm}$ by NTA. The yield of EV will depend on factors such as the liver weight and losses within the perfusate or ultracentrifugation steps.

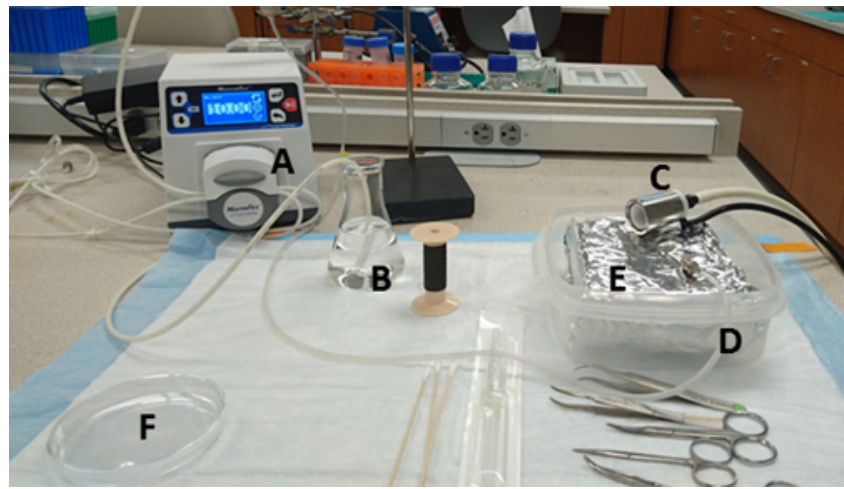

Figure 1: Bench preparation. The materials and their locations are: (A) pump, (B) warmed $125 \mathrm{~mL}$ flask containing HBSS and water suction port, (C) nose cone connected to an Isoflurane vaporiser, (D) water exhaust port of the pump connecting with needle, (E) tray lined with aluminum foil inside a hard walled container, and $(F) 10 \mathrm{~cm}$ culture dish in which collagenase medium is poured in advance. Please click here to view a larger version of this figure. 

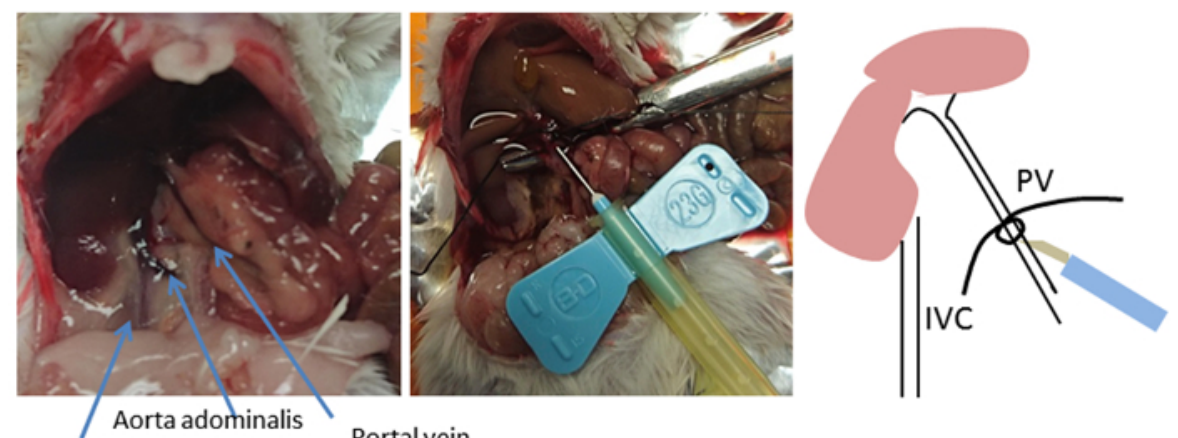

Inferior vena cava

Portal vein

Figure 2: Cannulation site. The anatomy of the mouse abdomen is shown. Using curved forceps, a thread is placed underneath the portal vein (PV) and a loose knot is tied. The insertion location is near the liver, $5-10 \mathrm{~mm}$ below the ligature, but not beyond the first portal branch (left and right hepatic portal veins). The cannula is fixed or fastened using thread with a stopper knot. This knot serves as a marker of the PV location if the cannula is dislodged. Please click here to view a larger version of this figure.

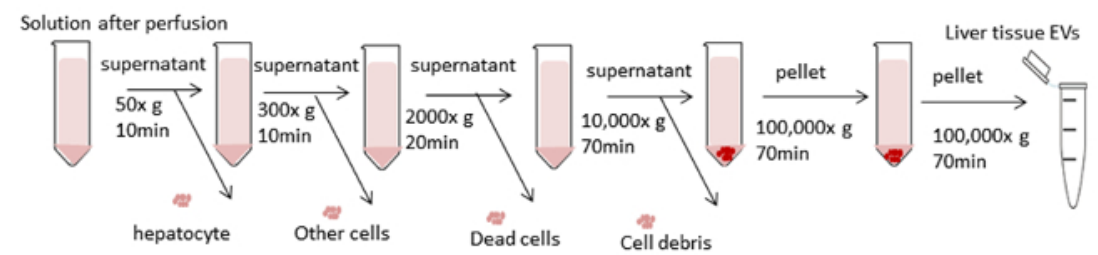

Figure 3: Schematic of centrifugation steps. The goal is to remove unwanted cells and other components and isolate EVs. The first four centrifugation steps are designed to remove hepatocytes and other cells, dead cells, or cell debris using differential centrifugation. After these steps, ultracentrifugation is performed at $100,000 \times \mathrm{g}$ to collect the pellet of EVs. The pellet is washed by re-suspending in PBS and subjected to a final ultracentrifugation at $100,000 \times \mathrm{g}$. All the centrifugation steps are carried out at $4{ }^{\circ} \mathrm{C}$. Please click here to view a larger version of this figure.

A

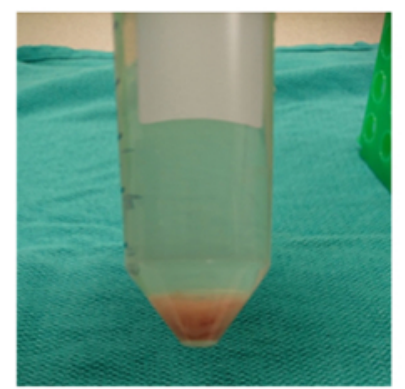

C

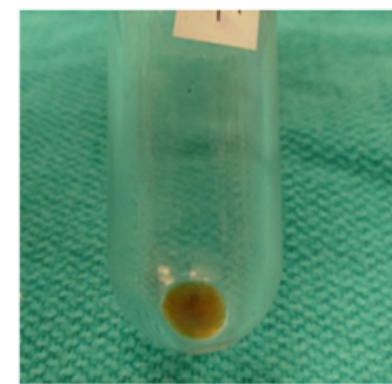

B

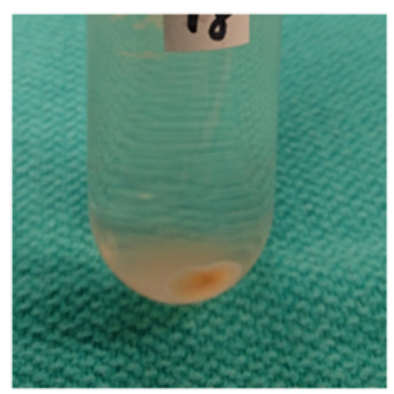

D

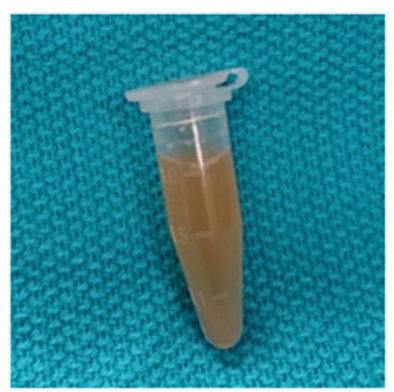

Figure 4: Differential centrifugation. (A) After centrifugation at $50 \mathrm{xg}$ for $10 \mathrm{~min}$, a pellet containing hepatocytes is observed. (B) A roundbottom tube is used for centrifugation at $10,000 \times \mathrm{g}$ for $70 \mathrm{~min}$ to remove cell debris. (C) A polycarbonate ultracentrifuge tube is used for centrifugation at $100,000 \times \mathrm{g}$ for $70 \mathrm{~min}$. The pellet is collected in one tube and washed by re-suspending with PBS. (D) The final pellet is resuspended in $1000 \mu \mathrm{L}$ of PBS. Please click here to view a larger version of this figure. 


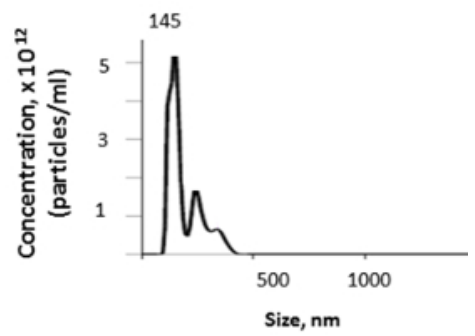

Figure 5: Representative result. The size and concentration of liver tissue EVs can be determined by nanoparticle tracking analysis (NTA). Please click here to view a larger version of this figure.

\section{Discussion}

This protocol describes an optimal and reproducible method for the isolation of hepatic tissue EV using a two-step perfusion process via the portal vein followed by differential ultracentrifugation. Important steps of the procedure include cannula placement, collagenase concentration and digestion time, flow speed of the medium, handling of the tissue after digestion, and classical differential ultracentrifugation.

Cell separation is achieved by separation from connective tissue components after digestion using collagenase type IV.The concentration of collagenase used for perfusion can range from 0.1 to $5 \mathrm{mg} / \mathrm{mL}$. There can be considerable batch-to-batch variation in the efficacy of collagenase for tissue digestion. Concentrations of collagenase from 0.5 to $5 \mathrm{mg} / \mathrm{mL}$ were tested, but the concentration used did not have a major impact on the yield of EVs obtained. Using a higher concentration of collagenase will result in a more rapid swelling and whitening of the liver. The goal is to obtain satisfactory cell dissociation without excessive contamination or damage. An optimal concentration of collagenase used in these isolations is $1-2 \mathrm{mg} / \mathrm{mL}$ perfused for $7-8 \mathrm{~min}$ at a flow rate of $8 \mathrm{~mL} / \mathrm{min}$. A perfusion procedure that is too long will increase the risk of destroying the thin connective tissue within the liver as well as increase technical risks such as needle dislodgment from the portal vein or air trapping with the vein.

The most challenging aspect of this protocol is the cannulation of the portal vein. This can be challenging to perform, particularly in mice in the 18- to 25-g size range. The techniques for collagenase perfusion were originally developed for use in rats and subsequently adopted for use in mice after numerous modifications and adjustments. Cannulation using a $23 \mathrm{G}$ blood collection set is easier than placement of a catheter in blood vessels of small luminal diameter. Fixing the cannula using thread with a stopper knot is recommended to avoid dislodgement and the knot also serves as a marker of portal vein location in case the cannula comes off the vessel.

For downstream analysis, it is extremely important to have minimal contamination from cells. There are several important considerations in the handling of tissue after digestion. First, the forceps and scissors are changed when the liver is extracted to avoid blood contamination. Second, it is critical that the gallbladder be carefully removed from the liver to avoid tearing and unwanted contamination from bile. Third, once the liver has been removed from the mouse, the liver is washed very gently using PBS to remove any blood. Minimizing contamination with cells should be given a higher priority than reduction in the yield of EVs obtained.

Ultracentrifugation is the most commonly used method for the isolation and purification of $E V s^{8,9,10,11}$. This approach will remove most parenchymal cells such as hepatocytes or cholangiocytes and non-parenchymal cells such as Kupffer cells, sinusoidal endothelial cells, and stellate cells, In addition, cell debris, cell aggregations, and dead cells will also be removed by differential centrifugation. Further purification and isolation of specific populations can be performed by size exclusion chromatography to remove any non-vesicular protein aggregates or lipoproteins.

A limitation of this protocol is that it may not capture all tissue vesicles, given the possibility that some vesicles may be removed in the perfusate. If a global assessment is needed, collection of perfusate and isolation of vesicles within perfusate should be considered. A further limitation is the potential for cell damage. To monitor the potential impact of excessive cell death, cell viability can be monitored and incorporated within quality parameters for tissue EV isolations. In conclusion, this procedure describes an optimized workflow using a two-step perfusion technique via the portal vein followed by differential ultracentrifugation for obtaining liver tissue EVs from mouse livers at a high yield. These tissue EVs are suitable for downstream analyses such as characterization of biomolecular composition and other studies that aim to characterize their physiological or pathophysiological roles or potential applications as disease markers.

\section{Disclosures}

The authors have nothing to disclose.

\section{Acknowledgments}

This study was supported by funding from National Cancer Institute Grant CA-217833. 


\section{References}

1. Kogure, T., Lin, W. L., Yan, I. K., Braconi, C., Patel, T. Intercellular nanovesicle-mediated microRNA transfer: a mechanism of environmental modulation of hepatocellular cancer cell growth. Hepatology. 54 (4), 1237-1248 (2011).

2. Maji, S., Matsuda, A., Yan, I. K., Parasramka, M., Patel, T. Extracellular vesicles in liver diseases. American Journal of Physiology Gastrointestinal and Liver Physiology. 312 (3), G194-G200 (2017).

3. Kogure, T., Patel, T. Isolation of extracellular nanovesicle microRNA from liver cancer cells in culture. Methods in Molecular Biology. 1024, 11-18 (2013).

4. Miller, L. L., Bly, C. G., Watson, M. L., Bale, W. F. The dominant role of the liver in plasma protein synthesis; a direct study of the isolated perfused rat liver with the aid of lysine-epsilon-C14. Journal of Experimental Medicine. 94 (5), 431-453 (1951).

5. Seglen, P. O. Preparation of isolated rat liver cells. Methods in Cellular Biology. 13, 29-83 (1976).

6. Klaunig, J. E., et al. Mouse liver cell culture. I. Hepatocyte isolation. In Vitro. 17 (10), 913-925 (1981).

7. Li, W. C., Ralphs, K. L., Tosh, D. Isolation and culture of adult mouse hepatocytes. Methods in Molecular Biology. 633 185-196 (2010).

8. Yin, Z., Ellis, E. C., Nowak, G. Isolation of mouse hepatocytes for transplantation: a comparison between antegrade and retrograde liver perfusion. Cell Transplantation. 16 (8), 859-865 (2007).

9. Thery, C., Amigorena, S., Raposo, G., Clayton, A. Isolation and characterization of exosomes from cell culture supernatants and biological fluids. Currents Protocols in Cell Biology. Chapter 3 (Unit 3.22), (2006).

10. Raposo, G., et al. B lymphocytes secrete antigen-presenting vesicles. Journal of Experimental Medicine. 183 (3), 1161-1172 (1996).

11. Witwer, K. W., et al. Standardization of sample collection, isolation and analysis methods in extracellular vesicle research. Journal of Extracellular Vesicles. 2, (2013). 